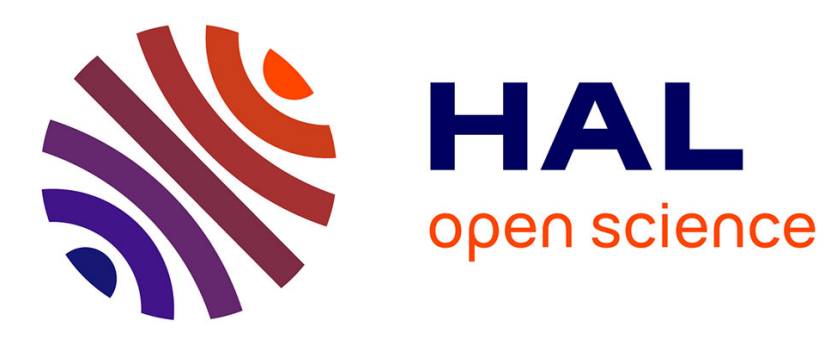

\title{
Nonlinear Control for Urban Vehicles Platooning, Relying upon a Unique Kinematic GPS
}

Jonathan Bom, Benoît Thuilot, François Marmoiton, Philippe Martinet

\section{To cite this version:}

Jonathan Bom, Benoît Thuilot, François Marmoiton, Philippe Martinet. Nonlinear Control for Urban Vehicles Platooning, Relying upon a Unique Kinematic GPS. 2005 IEEE International Conference on Robotics and Automation, Apr 2005, Barcelona, Spain. pp.4138-4143, 10.1109/ROBOT.2005.1570755 . hal-02467166

\section{HAL Id: hal-02467166 https://hal.inria.fr/hal-02467166}

Submitted on 5 Feb 2020

HAL is a multi-disciplinary open access archive for the deposit and dissemination of scientific research documents, whether they are published or not. The documents may come from teaching and research institutions in France or abroad, or from public or private research centers.
L'archive ouverte pluridisciplinaire HAL, est destinée au dépôt et à la diffusion de documents scientifiques de niveau recherche, publiés ou non, émanant des établissements d'enseignement et de recherche français ou étrangers, des laboratoires publics ou privés. 


\title{
Nonlinear Control for Urban Vehicles Platooning, Relying upon a Unique Kinematic GPS
}

\author{
J. Bom, B. Thuilot, F. Marmoiton and P. Martinet \\ LASMEA - 24 avenue des Landais - 63177 AUBIERE - FRANCE \\ bom@lasmea.univ-bpclermont.fr
}

\begin{abstract}
Index Terms - mobile robots, nonlinear control, platooning, Automatic Guided Vehicles, RTK GPS.

Abstract-In order to solve problems of traffic saturation in cities, new alternative "Urban Transportation Systems" are based on electric vehicles in free-access. One necessary functionality of such systems is their ability to move in a platoon fashion. Platooning of these automatic guided vehicles, relying on RTK-GPS sensors and inter-vehicles communication, is addressed in this paper. More precisely, vehicles platoon is expected to follow a curved reference path. Relying on nonlinear control theory, lateral and longitudinal control are fully decoupled, and therefore addressed independently. To ensure passengers comfort, additional monitoring functions supervise our control system. Then, simulations followed by experiments carried out with urban vehicles, are presented.
\end{abstract}

\section{INTRODUCTION}

Nowadays, congestion of vehicles traffic in cities, pollution that is linked, and people security problems are current and important concerns. As a consequence, new alternative transport solutions must be found. Numerous researches are addressed in large projects so-called "Urban Transportation Systems". Urban electric vehicles, available for example in a car-sharing concept, seem to be an interesting solution. On one hand, they reduce considerably the cited nuisances; and on the other hand, they propose a great flexibility, which could satisfy users. Then, their potential applications appear wide: they appear welcomed in urban structure, where they can be used in the transports of goods, in public transport, to visit attraction resorts, etc. Such systems have been developed since the mid-90's, e.g. Praxitèle in France [9]-[2], CarLink in the USA [16], Crayon in Japan [5]...

In order to transport many passengers, to drive vehicles along main urban roads, to bring back vehicles to their base station, etc., urban mobile robots with fully automated driving capabilities must be able to move in a platoon fashion. Our research interest is platooning of vehicles composed of a leader followed by vehicles in a single file. To confer autonomy to a platoon, it is necessary to localize accurately the automatic guided vehicles in their environment. Some applications, requiring equipped infrastructures, are in developing or developed: automatic vans in suspension over a guideway, thanks to Electro Magnetic forces, are described in [11], fleet of urban shuttles detecting magnetic track integrated in the road pavement is described in [6]. Unluckily, the cost of implementing the infrastructure and the fact that these systems can not be used outside of the equipped area appear restricted. RTK GPS (Real Time Kinematic Global Positioning System) sensors do not present these drawbacks, and since they can provide in realtime vehicle localization with a centimeter accuracy, they appear very attractive, at least for guiding the leader vehicle. Nowadays RTK GPS sensors seem to be expensive and risks of canyoning effect in urban environment is real. However, the evolution of car equipment market and a wider satellites cover thanks to EGNOS and Galileo constellations, are going to reduce the costs and rise up the performances of that sensor.

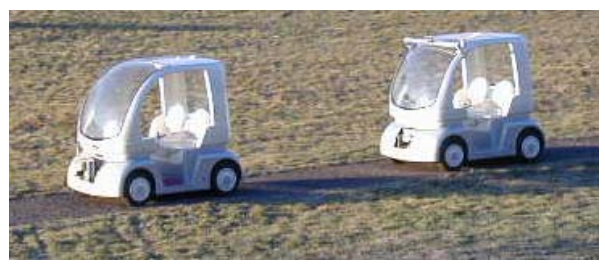

Fig. 1. Our experimental vehicles : Cycabs

In platooning applications, relative distance and speed with respect to the preceding vehicle are also needed. Such information could be provided by direct sensors (as cameras [3], radar [8], ...), which also do not require an equipped environment. However drawbacks of such sensors are their field of vision too small when platoons describe reference paths with high curvature, and the imperious necessity that no obstacle is present between the vehicles of the platoon. To overcome such problems, a conceivable solution is that vehicles share absolute localization measurements provided by their own RTK-GPS sensor via inter-vehicles communication. Relevancy of this approach is demonstrated in this paper. Urban electric vehicles called Cycabs (Fig1), which serve as development products in several French laboratories, have been used, and intervehicles communication relies on WiFi technology. In the forthcoming experimental results, the leader vehicle is driven automatically, and each vehicle in the platoon follows the same reference path. Nevertheless, since intervehicles communication is available, it could also be possible that the leader vehicle was driven manually and that the other vehicles objective was to follow leader vehicle evolution.

Some results in vehicles platoon control relying on RTK GPS can be found in the literature. In [1], each vehicle in the platoon is equipped with two GPS sensors in order to determine precisely vehicle location and heading. The reference path for this three-vehicles platoon is defined by 
the evolution of the first vehicle. The platoon is operating at a constant speed fixed between $20 \mathrm{~km} / \mathrm{h}$ and $90 \mathrm{~km} / \mathrm{h}$ and the control objective is to maintain a safe driving distance of approximatively $2 s$. Reported experiments present joining, leaving and passing the platoon. In [13], an automatic following device, making use of GPS and vehicleto-vehicle communication, enables an unmanned vehicle to follow a driven leading one. These works are more developed upon the technological sensor aspects (GPS and communication respectively) rather than upon control design ones, contrarily to the work that is presented here.

Since urban applications are addressed, vehicles velocities between $10 \mathrm{~km} / \mathrm{h}$ and $20 \mathrm{~km} / \mathrm{h}$ are considered (this is exactly the velocity range of Cycab vehicles). The objective in platooning is to control vehicles velocity in order to respect some constraints: generally a constant direct distance or time between the cars is regulated (see e.g. [7]). In this paper, the platooning objective is to keep a constant curvilinear distance within vehicles. The main advantage of curvilinear distance is that it agrees with the distance travelled (monotonous behavior) and is perfectly consistent when following reference paths with high curvature (which is not the case with direct distance). Platooning control design relies on nonlinear techniques, as in [4], instead of control approaches based on linear approximations (e.g. [12] [13]). Since no approximation is achieved, performances provided by the nonlinear control law are more satisfactory and more robust than those offered by linear control. Moreover, this control approach allows to fully decouple longitudinal and lateral controls. In [17], automatic lateral guidance has been presented for one vehicle. Thanks to this decoupling feature, lateral guidance of each vehicle in the platoon can indeed be achieved independently, by relying on that control law. Therefore, the present paper addresses only longitudinal control. Finally, in order to ensure passengers security and comfort, a monitoring approach is set to manage saturations of the nonlinear platooning control law.

The paper is organized as follows: first our experimental platform is described. Then, vehicle modelling is addressed. After that, platoon control law design is presented. Simulation, and finally full-scale experiments are then reported.

\section{EXPERIMENTAL VEHICLE}

In this section, RTK GPS receiver and experimental vehicles constituting the platoon are successively presented.

The RTK GPS receiver is a "Sagitta" unit from Thales Navigation. It provides position measurements at a $10 \mathrm{~Hz}$ sampling frequency, with a $2 \mathrm{~cm}$ accuracy. The GPS reference station has been located on a $15 \mathrm{~m}$-height building. Each Cycab is equipped with a unique GPS sensor located on the roof of the vehicle straight up the mid-distance between the rear wheels. The radio receiver is in front of the vehicle.

Experimental vehicles, named Cycab, are depicted on fig1. Two passengers can be transported simultaneously. They have been designed specifically for car-free cities.
Indeed, the small dimensions (length : $1.90 \mathrm{~m}$, width : $1.20 \mathrm{~m})$ are advantages in urban traffic. These vehicles are entirely under control computer. They can be driven manually with a joystick, or automatically. Cycabs have two hours autonomy, and the used kinematic configuration is the same as a car-like vehicle. Its maximum speed is $18 \mathrm{~km} / \mathrm{h}$. In our experiments, a personal computer is added in each Cycab. This high-level computer collects information received from GPS, mounted by serial link, and from front vehicles, via $\mathrm{WiFi}$. The steering values and the vehicle velocities computed by control laws are transmitted to the Cycab computer by an Ethernet network and reach the two MPC555 micro-controllers (one for front wheels, the other one for rear wheels) by CAN.

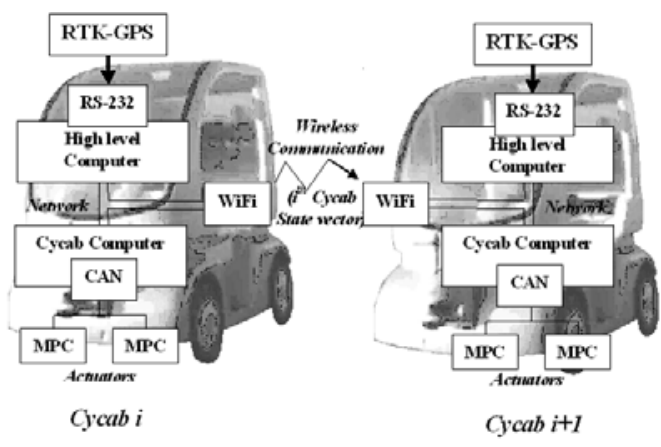

Fig. 2. Architecture

\section{PlatoON MODELING}

\section{A. Modeling Assumptions}

Since Cycabs are devoted to move in urban environments, i.e. at low speed on asphalted grounds, sliding effects can be disregarded (as corroborated by extensive experiments). Therefore, it is consistent to design control laws from a vehicle kinematic model. Hereafter, the celebrated tricycle model, validated by numerous laboratories [10] [15] [3] [17], has been used: the two actual front wheels are replaced by a unique virtual wheel located at the mid-distance between the actual wheels. The notations used are detailed and illustrated in fig 3:

- $C$ is the common reference path, defined in an absolute frame $\left[A, X_{A}, Y_{A}\right)$.

- $O_{i}$ is the center of the $i^{\text {th }}$ vehicle rear axle.

- $M_{i}$ is the closest point on $C$ to $O_{i}$.

- $s_{i}$ is the curvilinear coordinate of point $M_{i}$ along $C$, $c\left(s_{i}\right)$ denotes the curvature of path $C$ at this point, and $\theta_{c}\left(s_{i}\right)$ stands for the orientation of the tangent to $C$ at $M_{i}$, with respect to frame $\left[A, X_{A}, Y_{A}\right)$.

- $\theta_{i}$ is the heading of $i^{\text {th }}$ Cycab at point $O_{i}$, with respect to frame $\left[A, X_{A}, Y_{A}\right)$.

- $\tilde{\theta}_{i}=\theta_{i}-\theta_{c}\left(s_{i}\right)$ denotes the angular deviation of the $i^{\text {th }}$ vehicle with respect to $C$.

- $y_{i}$ is the lateral deviation of the $i^{t h}$ vehicle with respect to $C$.

- $\delta_{i}$ is the orientation of the $i^{t h}$ vehicle front wheel with respect to its centerline. 
- $l$ is Cycab wheelbase.

- $v_{i}$ is the $i^{t h}$ vehicle linear velocity at point $O_{i}$.

- $n$ is the number of vehicles in the platoon.

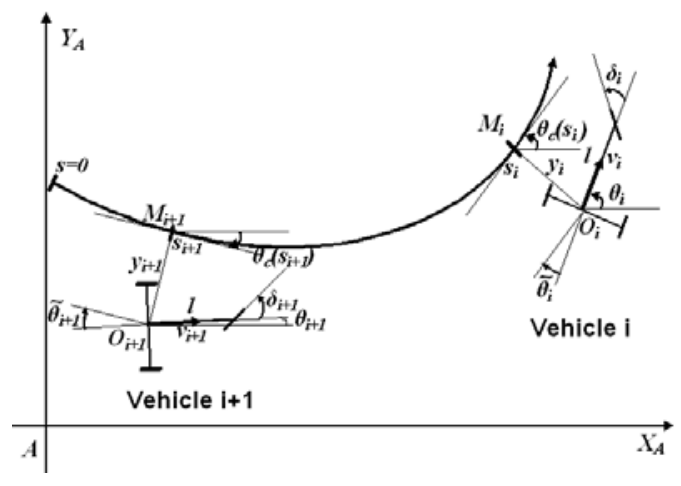

Fig. 3. Model tricycle description

\section{B. State Space Model Derivation}

The vector $\left(s_{i}, y_{i}, \tilde{\theta}_{i}\right)$ describes the state of the $i^{t h}$ vehicle. The celebrated tricycle model is (see [15], [3], [17]):

$$
\begin{gathered}
\dot{s}_{i}=v_{i} \frac{\cos \tilde{\theta}_{i}}{1-y_{i} c\left(s_{i}\right)} \\
\dot{y}_{i}=v_{i} \sin \tilde{\theta}_{i} \\
\dot{\tilde{\theta}}_{i}=v_{i}\left(\frac{\tan \delta_{i}}{l}-\frac{c\left(s_{i}\right) \cos \tilde{\theta}_{i}}{1-y_{i} c\left(s_{i}\right)}\right)
\end{gathered}
$$

Control objectives are to bring and maintain $y_{i}$ and $\tilde{\theta}_{i}$ to 0 , thanks to $\delta_{i}$, and $\left(s_{i}-s_{i+1}\right)$ to a fixed value, thanks to $v_{i}$. State variables $s_{i}$ and $y_{i}$ are directly provided by GPS sensor. $\tilde{\theta}_{i}$ could a priori be inferred from vehicle velocities, also provided by GPS sensor, but velocities measurements are too noisy, so that $\tilde{\theta}_{i}$ has to resort to a Kalman reconstructor, see [17] for more details.

\section{Control Law Design}

First, it is shown that longitudinal and lateral controls can be decoupled. Then longitudinal control law is designed. Moreover, it is considered that : $y_{i} \neq \frac{1}{c\left(s_{i}\right)}$ (vehicle is not on the reference path curvature center) and $\tilde{\theta}_{i} \neq \frac{\pi}{2}[\pi]$. In practical situations, if the $n$ vehicles are well initialized, such difficulties never arise.

\section{A. Decoupling feature}

Via invertible state and control transformations, nonlinear Cycab model (1)-(2)-(3) can be converted, in an exact way, into the following so-called chained form, see [15]:

$$
\begin{aligned}
& \dot{a}_{1 i}=m_{1 i} \\
& \dot{a}_{2 i}=a_{3 i} m_{1 i} \\
& \dot{a}_{3 i}=m_{2 i}
\end{aligned}
$$

where $A=\left(a_{1 i}, a_{2 i}, a_{3 i}\right)^{T}=\Theta\left(s_{i}, y_{i}, \tilde{\theta}_{i}\right)$ is the chained state vector and $M=\left(m_{1 i}, m_{2 i}\right)^{T}=\Upsilon\left(v_{i}, \delta_{i}\right)$ is the chained control vector. From this chained form, a large part of linear systems theory can be used (but, since the transformations are exact, it is not required that vehicle configuration is in a specific configuration, contrarily to tangent linearization techniques). More precisely, it can be noticed that lateral control (i.e. control of $a_{2 i}$ and $a_{3 i}$ ) can be achieved by designing only $m_{2 i}$. Since it can be shown that $m_{2 i}$ is related in an invertible way to $\delta_{i}$ (provided that $v_{i} \neq 0$ ), lateral control is fully decoupled from longitudinal one: in lateral control, $v_{i}$ appears as a free parameter, that can now be used to achieve longitudinal control. Details and performances of lateral control are presented in [17].

\section{B. Longitudinal Control}

1) Standard Mode: Longitudinal (or platooning) control aims at preserving a constant curvilinear distance $d$, between the $i^{t h}$ and the $(i+1)^{t h}$ vehicles. So, the control objective is to keep $e_{i}$ equal to 0 , with

$$
e_{i}=\left(s_{i}-s_{i+1}\right)-d
$$

When differentiating $e_{i}$, it can be obtained that:

$$
\dot{e}_{i}=\frac{v_{i} \cos \tilde{\theta}_{i}}{1-y_{i} c\left(s_{i}\right)}-\frac{v_{i+1} \cos \tilde{\theta}_{i+1}}{1-y_{i+1} c\left(s_{i+1}\right)}
$$

Just as in lateral control design, exact linearization techniques can also be used: the actual control variable is the follower velocity $v_{i+1}$. Let us however introduce auxiliary control $u_{i+1}$, related to $v_{i+1}$ via:

$$
v_{i+1}=\frac{1-y_{i+1} c\left(s_{i+1}\right)}{\cos \tilde{\theta}_{i+1}}\left(\frac{v_{i} \cos \tilde{\theta}_{i}}{1-y_{i} c\left(s_{i}\right)}-u_{i+1}\right)
$$

Error evolution is then simply given by $\dot{e}_{i}=u_{i+1}$. Therefore, it is intuitive to design the auxiliary control law as:

$$
u_{i+1}=-k e_{i}
$$

with $(k>0)$. Reporting (7) in (6) provides us with the actual platoon control law:

$$
v_{i+1}=\frac{1-y_{i+1} c\left(s_{i+1}\right)}{\cos \tilde{\theta}_{i+1}}\left(\frac{v_{i} \cos \tilde{\theta}_{i}}{1-y_{i} c\left(s_{i}\right)}+k e_{i}\right)
$$

Longitudinal control performances can then be adjusted at will by tuning gain $k$. The desired gain value, denoted $k_{\max }$, has however to be altered when longitudinal error $e_{i}$ is large, in order to guarantee that control variable $v_{i+1}$ always meets the application constraints :

$$
0 \leq v_{i+1} \leq v_{\max }
$$

(i.e. the follower vehicle is not supposed to go backward even if $e_{i}$ is largely negative, nor to climb beyond a prespecified maximum velocity $v_{\max }$ even if $e_{i}$ is largely positive). Therefore, control gain $k_{\max }$ is here decreased according to the smooth adaptive gain tuning depicted on fig 4. Straightforward computations can display that constraints (9) are then satisfied, at least in standard situations where lateral errors are small (i.e. when $\frac{1-y_{i+1} c\left(s_{i+1}\right)}{\cos \tilde{\theta}_{i+1}} \approx 1$ and $\left.\frac{\cos \tilde{\theta}_{i}}{1-y_{i} c\left(s_{i}\right)} \approx 1\right)$. 


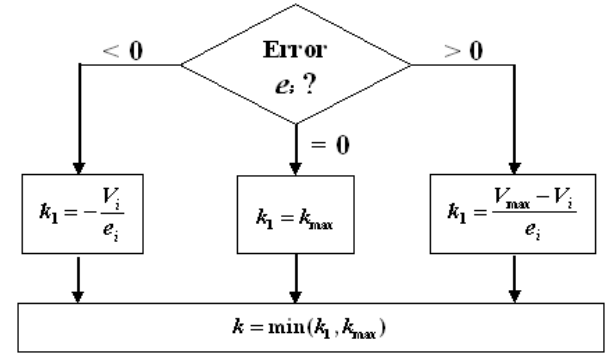

Fig. 4. Adaptive gain tuning

2) Monitoring: Longitudinal control law (8), together with the adaptive gain tuning shown on fig 4, ensures that $v_{i+1}$ belongs to the acceptable velocity range (9). However, vehicle acceleration is not upper-bounded, which may be unpleasant to passengers. Therefore, a monitoring scheme, shown on fig 5, has been introduced to take care of passengers comfort, as far as their security is guaranteed.

More precisely, the notation $a_{\text {comf }}$ on Figure 5 stands for the maximum acceleration/deceleration comfortable to a passenger aboard. Then, if the acceleration/deceleration of vehicle $i+1$, denoted $a_{i+1}$, is superior to that value, a security test is performed, in order to investigate if $a_{i+1}$ can or cannot be safely limited to $\pm a_{\text {comf }}$ :

- the case $a_{i+1}>a_{\text {comf }}>0$ may occur if vehicle $i+1$ is far behind vehicle $i$. In such a situation, $a_{i+1}$ can be limited to $a_{\text {comf }}$ without any collision risk.

- the case $a_{i+1}<-a_{\text {comf }}<0$ may occur if vehicle $i$ slows down abruptly. The final distance $\hat{d}_{i}$ between vehicle $i+1$, if it was stopped with a deceleration $-a_{\text {comf }}$, and vehicle $i$, if it was remaining immobile, is then computed.

$\rightarrow$ if $\hat{d}_{i}$ is superior to a security distance $d_{\text {secur }}$, then $a_{i+1}$ can also be safely limited to $-a_{\text {comf }}$.

$\rightarrow$ if $\hat{d}_{i}<d_{\text {secur }}$, the urgency deceleration $-a_{\text {urg }}<$ $-a_{\text {comf }}$ leading to a final distance equal to $d_{\text {secur }}$ is computed, and $a_{i+1}$ is limited to that value. Passengers security is therefore guaranteed.

When computing $\hat{d}_{i}$ and $a_{\text {urg }}$, delays introduced by actuator features and transmission latencies are considered.

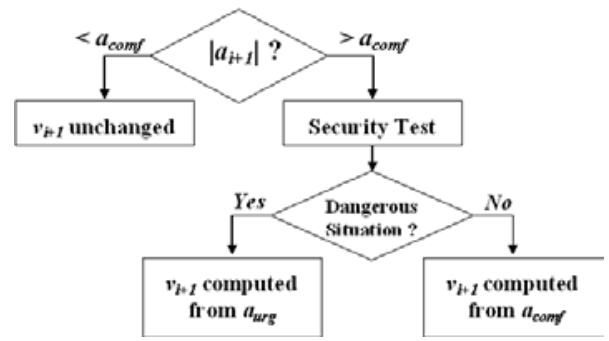

Fig. 5. Monitoring scheme

\section{Simulations}

In simulations, gains of the lateral control law are tuned to impose that lateral deviation converges to 0 within $15 \mathrm{~m}$. Gains of the longitudinal control law are set to $k_{\max }=0.6, v_{\max }=4 \mathrm{~m} / \mathrm{s}$ and $a_{\text {comf }}=1 \mathrm{~m} / \mathrm{s}^{2}$. This last value is ensued from studies reported in [18]. The desired curvilinear distance is chosen equal to $8 \mathrm{~m}$ and $d_{\text {secur }}$ is set equal to $3 \mathrm{~m}$. Finally RTK-GPS sensor features are introduced in simulation via a white noise with a standard deviation of $2 \mathrm{~cm}$ added to position measurements.

Simulation results are reported on fig 6 to 9 . Vehicles are initially parked besides the reference path. The objective is: when the leader arrives close to the second vehicle, it hooks it. Then, the second vehicle hooks the third one and so on up to the $6^{\text {th }}$ one.

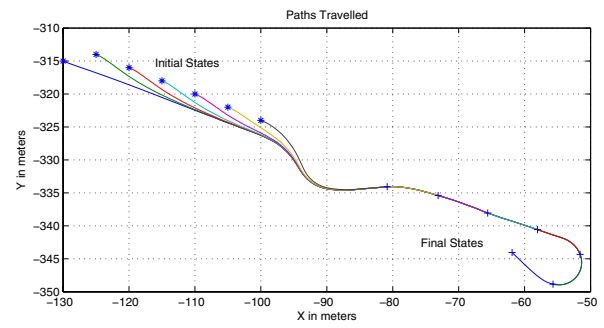

Fig. 6. Path following achieved by the platoon

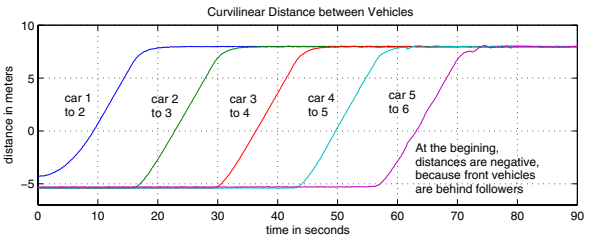

Fig. 7. Curvilinear distances during platoon formation

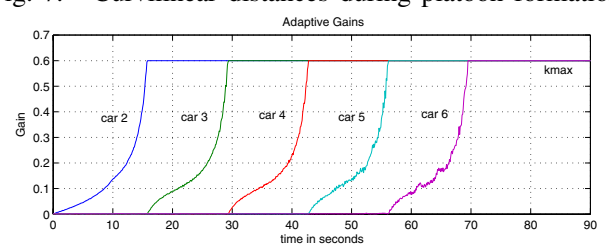

Fig. 8. Adaptive gain evolution

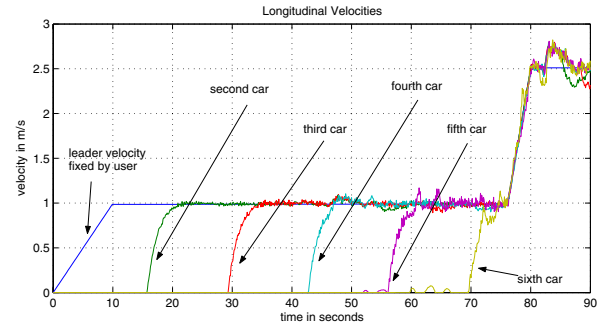

Fig. 9. Longitudinal velocities during platoon formation

The leader velocity is specified by the user : it starts from $0 \mathrm{~m} / \mathrm{s}$ and reaches progressively $1 \mathrm{~m} / \mathrm{s}$. During platoon formation, the influence of adaptive gain can be observed: the more the error is important, the smaller the gain is. By this way, vehicles velocities remain also inferior to $v_{\max }$, as desired. Once the platoon has been constituted, (fig7 and fig9), it can be noticed that each longitudinal error tends to zero, and consequently, gain $k$ reaches its maximal value. At time $t=75 \mathrm{~s}$, leader velocity is increased up to $2.5 \mathrm{~m} / \mathrm{s}$. Velocities of the follower, as expected, stay close 
to the leader one (fig9). However, limitations of near-tonear approach (only information relative to the preceding vehicle are used to compute $v_{i+1}$ ) can be visualized: prior to $t=75 \mathrm{~s}$, velocity evolutions of the last followers are much more noisy than the evolutions of the first ones, due to errors accumulation from vehicle to vehicle. Therefore, it appears utopian to control a platoon composed of numerous vehicles with a control law based on a near-to-near approach, which can only be seen as a first step in platoon control design. Finally, it can be observed that reference path curvature has no influence on vehicles velocities, and inter-distances. This was expected from lateral/longitudinal decoupling features exhibited by chained form model.

\section{EXPERIMENTAL RESUlts}

Several experiments have been carried out at "Campus des Cézeaux", in Clermont-Ferrand area. The experiments presented here involve only two Cycabs. However, experiments with more vehicles are planned at short term, since our lab will soon be equipped with additional vehicles.

The reference path is shown on fig 10 . The desired vehicle inter-distance and the values of the control gains are chosen identical to those described in the simulation section. The first experiment presents a hooking, and then the control law (in standard mode described by (8)) maintains the desired gap. At initial time, the first Cycab is close to the reference path and the second is waiting for the passage of the leader, see fig 10 . The leader velocity is $1 \mathrm{~m} / \mathrm{s}$. Accuracy of the lateral control law is very satisfactory: the maximum lateral deviation is close to $3 \mathrm{~cm}$ during straight lines and $10 \mathrm{~cm}$ during curves. This is in accordance with lateral performances presented in [17] and these small errors are due to some delays at the actuators level. Longitudinal behavior is also the one expected: the second vehicle is evaluating its curvilinear distance to the leader one, thanks to GPS and WiFi communication. Once the desired curvilinear distance is close to be reached, it starts progressively, hooks the leader and joins it on the reference path. The longitudinal deviation can be observed on fig 11. The convergence to the desired gap is progressive, thanks to adaptive gain, see fig12. Once the second vehicle is hooked, the gap between the two vehicles presents a satisfactory mean value inferior to $1 \mathrm{~mm}$ and a standard deviation of $4.8 \mathrm{~cm}$. We can also notice that the follower trajectory converges progressively to the reference one, see fig 10. There is no violent turning of the vehicle towards the closest point on the reference path. This was expected since the follower has to converge to the reference path within $15 \mathrm{~m}$, according to the lateral control gains.

In the second experiment, monitoring mode is investigated. Control parameters are still the same as previously. At the beginning, the longitudinal control law is in standard mode. Then, the leader vehicle stops abruptly. The security test shown on fig 5 ensures that the follower vehicle can be stopped safely with deceleration $-a_{\text {comf }}$. Therefore, follower decelerates at $-1 \mathrm{~m} / \mathrm{s}^{2}$ until it stops, see fig 15 . Due to the constant deceleration, velocity decreases linearly and the curvilinear distance parabolically. During this abrupt

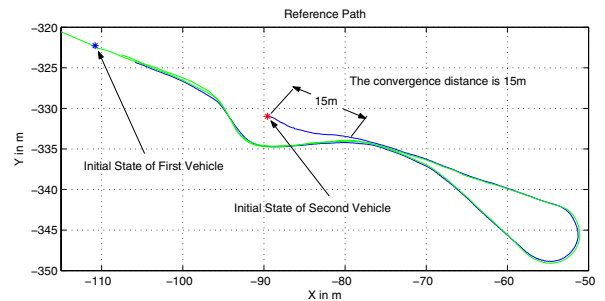

Fig. 10. Reference trajectory and initial states - First Experiment

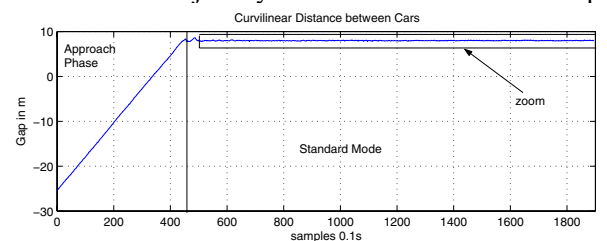

Fig. 11. Curvilinear distance between cars - first experiment

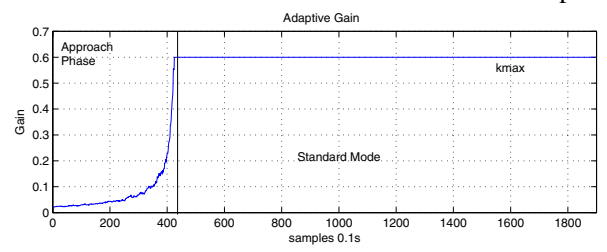

Fig. 12. Adaptive gain evolution - first experiment

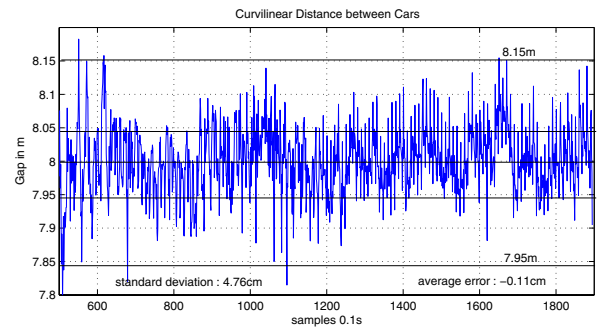

Fig. 13. Zoom on curvilinear distance - first experiment

stop, monitoring mode privileges passengers comfort instead of longitudinal performances. As a consequence, the braking distance is quite large, and the curvilinear distance is under the desired one. Finally, a delay between the instants when the leader stops, and when the follower begins to decelerate can be observed. It follows from actuation delays.

In the third experiment, security distance is raised up to $6.50 \mathrm{~m}$ instead of $3 \mathrm{~m}$ (others parameters are unchanged). In practical situations, we could imagine that the leader is pulling a trailer, and that collision occurs if the interdistance is inferior to $6.50 \mathrm{~m}$. Initially, the two Cycabs move at the same speed, with a constant gap close to $8 \mathrm{~m}$. Then, once more the leader stops abruptly. Security test depicted on fig 5 concludes that the follower vehicle cannot match $d_{\text {secur }}$ if it is stopped with deceleration $-a_{\text {comf } f}$. Therefore, an urgency deceleration equal to $-1.2 \mathrm{~m} / \mathrm{s}^{2}$ is computed and applied, see fig16 and fig 17.

When the two vehicles are stopped, it can be noticed that the curvilinear distance is $40 \mathrm{~cm}$ over $d_{\text {secur }}$. This just follows from the fact that actuator and transmission delays considered in $-a_{\text {urg }}$ computation are over-estimated. 


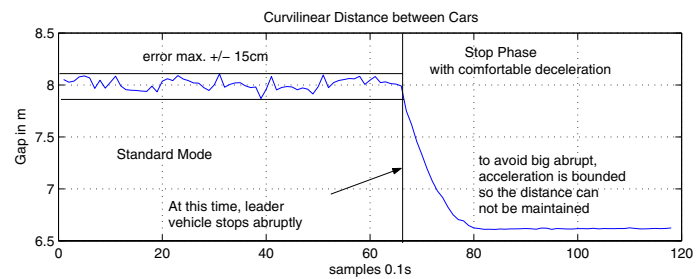

Fig. 14. Curvilinear distance between cars - Second Experiment

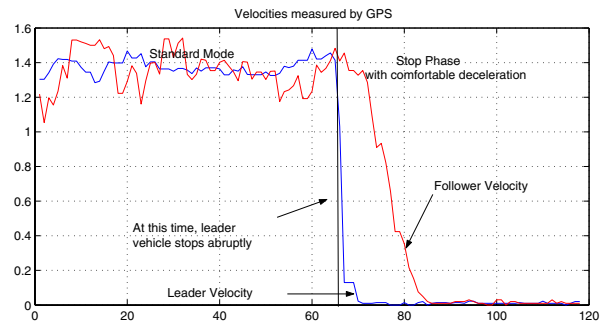

Fig. 15. Velocities - second experiment

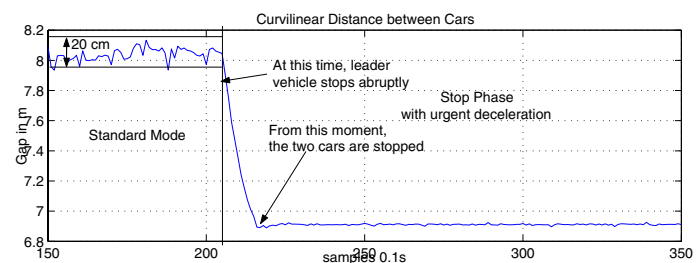

Fig. 16. Curvilinear distance between cars - third experiment

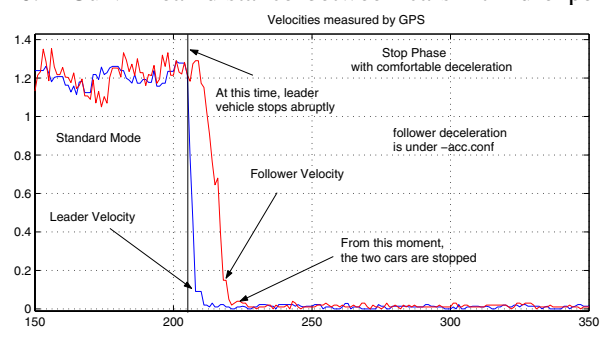

Fig. 17. Velocities - third experiment

\section{CONCLUSION}

In this paper, platooning control of automatic guided vehicles has been addressed. The case of urban electric vehicles, equipped with a RTK-GPS sensor, and exchanging data via WiFi communication, has been considered. Nonlinear control techniques have been used to fully decouple lateral and longitudinal control. Monitoring has been sketched in order to guarantee passengers security and comfort. Finally, performances of this control scheme are investigated via full-scale experiments.

Nevertheless, possible losses of signal satellites, due to canyoning effect in urban environment, are a major concern. To deal with this difficulty, data fusion combining GPS information (when available) to dead reckoning from inertial sensors is investigated to maintain continuously localization measurements. An other investigated solution consists to reconstruct the vehicles state by vision, see[14]. Finally, as seen in simulations, platoon stability with numerous vehicles can not be guaranteed in a satisfactory way as long as vehicles are controlled from near-to-near. We are presently considering the case where the follower receives information, not only from the preceding vehicle, but also from every vehicle that is ranked before it in the platoon. Control design is further investigated in order to benefit from these new information.

\section{ACKNOWLEDGMENT}

This work was funded by CPER Auvergne 2002-04 program.

\section{REFERENCES}

[1] M.E. Cannon, C. Basnayake, S. Crawford, S. Syed, and G. Lachapelle. Precise GPS sensor subsystem for vehicle platoon control. In Conf. ION GPS/GNSS, pages 213-224, Portland OR (USA), Sept. 2003.

[2] P. Daviet, S. Abdou, and M. Parent. Platooning for vehicles and automatic parking by scheduling robotic actions. In Intern. Symposium on Robotics and Manufacturing (WAC'96), Montpellier (France), 1996.

[3] P. Daviet and M. Parent. Platooning for small public urban vehicles. In $4^{\text {th }}$ Intern. Symposium Experimental Robotics (ISER'95), pages 345-354, Stanford, CA (USA), July 1995.

[4] A. De Luca, G. Oriolo, and C. Samson. Feedback control of a nonholonomic car-like robot. In Robot Motion Planning and Control, J.P. Laumond eds, volume 229 of Lectures Notes in Control and Information Sciences, pages 171-253, Springer-Verlag, 1998.

[5] P. Evans. New energy sources for the car : how toyota sees the future. Energy News (Australian Institute of Energy), 18(2), June 2000.

[6] D. Gillet and T. Chevroulet. BURST : Bright urban system for transportation. In $2^{\text {nd }}$ Intern. Workshop on European Scientific and Industrial Collaboration (WESIC'99), Newport (Wales), Sept. 1999.

[7] A. Girault and S. Yovine. Stability analysis of a longitudinal control law for autonomous vehicles. In IEEE Conf. on Decison and Control (CDC'99), volume 4, pages 3728-3733, Phoenix AR (USA), December 1999.

[8] H. Kuroda, S. Kuragaki, T. Minowa, and K. Nakamura. An adaptive cruise control system using a milimeter wave radar. In IEEE Intern. Conf. on Intelligent Vehicles (IV'98), volume 1, pages 168-172, Stuttgart (Germany), October 1998.

[9] C. Laugier. Towards autonomous vehicles for future intelligent transportation systems. In Proc. $6^{\text {th }}$ Conf. of Italian Association in Artificial Intelligence, pages 251-258, Padova (Italy), Sept. 1998.

[10] J.P. Laumond, editor. La Robotique Mobile. Hermes Science, Paris, Sept. 2001.

[11] $\mathrm{M}^{3}$ Team. The $\mathrm{M}^{3}$ urban transportation system. FTA Project Report MA-26-7077, MagneMotion Inc., Acton, MA (USA), January 2003.

[12] H. Makela, P. Kaarmila, and K. Koskinen. Convoy navigation. In $3^{\text {rd }}$ IFAC Conf. on Intelligent Autonomous Vehicles (IAV'98), pages 31-36, Madrid (Spain), March 1998.

[13] M. Ohtomo, R. Kimura, S . Fukushima, and N. Fujii. Automatic following system utilizing vehicle-to-vehicle communication. In IEEE Intern. Conf. on Intelligent Vehicles (IV'98), volume 2, pages 381-384, Stuttgart (Germany), October 1998.

[14] E. Royer, M. Lhuillier, M. Dhome, and T. Chateau. Towards an alternative GPS sensor in dense urban environment from visual memory. In British Machine Vision Conference, volume 1, pages 197-206, Kingston (England), September 2004

[15] C. Samson. Control of chained systems: application to path following and time-varying point stabilization of mobile robots. IEEE Trans. on Automatic Control, 40(1):64-77, January 1995.

[16] S. Shaheen, K. Wipyewski, C. Rodier, L. Novick, M.A. Meyn, and J. Wright. Carlink II: a commuter carsharing pilot program final report. PATH Research Report UCB-ITS-PRR-2004-23, Univ. of California, Berkeley (USA), August 2004.

[17] B. Thuilot, J. Bom, F. Marmoiton, and P. Martinet. Accurate automatic guidance of an urban electric vehicle relying on a kinematic GPS sensor. In $5^{\text {th }}$ IFAC Symposium on Intelligent Autonomous Vehicles (IAV'04), Lisboa (Portugal), July 2004.

[18] TranSafety Inc. Simulated on-the-road emergencies used to test stopping sight distance assumptions. Road Management and Engineering Journal, July 1997. 